\title{
Polymer Light Emitting Diodes with Doublet Emission
}

Qinying Gu, ${ }^{2}$ Alim Abdurahman, ${ }^{1}$ Richard H. Friend ${ }^{2 *}$ and Feng $\mathrm{Li}^{1^{*}}$

${ }^{1}$ State Key Laboratory of Supramolecular Structure and Materials, College of Chemistry, Jilin University, Qianjin Avenue 2699, Changchun, P. R. China;

2 Department of Physics, Cavendish Laboratory, Cambridge University, Cambridge CB3 OHF, UK;

${ }^{*}$ E-mail:

Prof. Feng Li: lifeng01@jlu.edu.cn

Prof. Sir Richard H. Friend: rhf10@cam.ac.uk 


\section{Experimental Methods}

\section{Thin-film preparation:}

The solid-state samples for steady-state UV-Vis, photoluminescence (PL) and temperature-dependent time-resolved PL measurements were prepared on the pre-cleaned Quartz substrates. PS-CzTTM-based films were spin-coated inside a solvent glovebox from anhydrous chlorobenzene solutions in either pristine or blended conditions. The samples were then annealed on the hot plate at $90^{\circ} \mathrm{C}$ for 10 minutes to remove the remaining solvent. The thickness of all the thin-films is ca. $80-100 \mathrm{~nm}$.

UV-VIS measurement and steady-state photoluminescence (PL) for solid-state samples:

The UV-vis absorption spectrum was measured by a Shimadzu UV-3600 Plus UV-VIS-NIR Spectrophotometer. Samples were measured under a transmission mode by using a photomultiplier tube (PMT) detector for the ultraviolet and visible regions. A blank substrate was used as a reference and the sample's absorbance was calculated by comparing the transmission of monochromated white light through the tested film and the transmission through the reference as a function of wavelength. PL spectra for the same samples were measured by an Edinburgh FLS980 Spectrometer. Samples were excited 
at $375 \mathrm{~nm}$ by a LED lamp and signals were captured by a single photon counting PMTs detector.

\section{Photoluminescent quantum efficiency (PLQE) measurement:}

Thin-film samples were placed inside an integrating sphere which was coated with diffusely reflective materials, ensuring all light inside the sphere are collected by the optical fibre connected to the detector. Samples were photoexcited by a continuous-wave (CW) laser at $405 \mathrm{~nm}$. Three measurements were taken for each sample: first is to directly excite the sample, second is not directly hitting the sample to subtract the PL generated by reflection or scattering and the last is to hit a blank Quartz substrate. All measurements were taken under nitrogen flow to avoid any oxygen quenching. The PLQE values were determined by using the method from De Mello et. al. ${ }^{1}$ Temperature-dependent time-resolved photoluminescence measurements (cryogenic PL measurement):

Solid-state samples were prepared as stated in Thin-film preparation. Timeresolved PL spectra were carried out by using an electrically-gated intensified CCD (ICCD) camera (AndoriStar DH740 CCI-010) coupled with an image identifier tube, giving high sensitivity and low noise level. A calibrated grating spectrometer (Andor SR303i) is connected to the ICCD for spectrum calibration. Photoexcitation is at the wavelength of $400 \mathrm{~nm}$, given by femtosecond laser pulses which were created by second harmonic generation (SHG) in a BBO crystal. A $425 \mathrm{~nm}$ long-pass filter was applied to avoid the 
scattered laser signals entering the camera. The kinetics of PL emissions can be obtained by setting the gate delay steps with respect to the excitation pulse. Liquid helium was used for sample cooling and a temperature-controlled cryostat was used for temperature regulation.

\section{Solution-processing device fabrication:}

Indium tin-oxide (ITO; WF 4.8 eV) coated glass substrates were subsequently cleaned by sonication in acetone and 2-propanol for 10 minutes, followed by $\mathrm{O}_{2}$ plasma treatment for 10 minutes. PEDOT: PSS (Clevios $\mathrm{CH} 4083$, LumTech Taiwan) was spin-casted on top of the ITO under ambient conditions and annealed on a hot plate at $160{ }^{\circ} \mathrm{C}$ for 20 minutes, forming a $40 \mathrm{~nm}$-thick film. The substrates were then transferred to a nitrogen-filled glovebox to conduct the following solution processes ${ }^{2}$. Poly(9-vinylcarbazone) (PVK, SigmaAldrich) as the hole injection layer (HIL) was spin-casted onto the PEDOT: PSS layer at the concentration of $15 \mathrm{mg} / \mathrm{ml}$ in toluene (Sigma-Aldrich) followed with an annealing process for $30 \mathrm{~min}$ at $150^{\circ} \mathrm{C}$. Annealed films were spin-rinsed with chlorobenzene (Sigma-Aldrich) to remove the residual soluble material and baked at $90^{\circ} \mathrm{C}$ for 10 minutes to remove the remaining rinsing solvent and form a 10-nm-thick rigid film. The $40 \mathrm{~nm}$-thick emitting layer (EML) of TPBi (LumTech Taiwan) doped with 10-15 wt.\% of PS-CzTTM (or host-free PS-CzTTM) in chlorobenzene was spin-coated on top of the PVK layer and baked on a hot plate at $90{ }^{\circ} \mathrm{C}$ for $10 \mathrm{~min}$ to remove any solvent present. The samples were then transferred to a vacuum deposition system. The $60 \mathrm{~nm}$-thick electron- 
transporting layer (ETL) (B3PYMPM, LumTech Taiwan), 0.8 nm-thick LiF (99.99\%, Sigma-Aldrich) and $100 \mathrm{~nm}$-thick aluminium were subsequently deposited by thermal evaporation under high vacuum $\left(<3 \times 10^{10-7} \mathrm{mbar}\right)^{2}$.

\section{Characterisation of OLED device performance:}

The forward-viewing current-voltage-luminance characteristics of these OLED devices were measured using a Keithley 2400 source meter, Keithley 2000 multimeter and a calibrated Si photodiode (from RS components), which was placed at a distance of $2 \mathrm{~cm}$ from the devices. External quantum efficiencies (EQE) were calculated from on-axis irradiance assuming a Lambertian emission profile and accounting for photodiode quantum efficiency across the electroluminescence spectrum. ${ }^{3}$ The electroluminescence spectra were obtained by a fibre spectrometer (Flame-S-VIS-NIR-ES, Ocean Optics). All the measurements were carried out at room temperature under ambient conditions.

\section{Time-resolved electroluminescence measurement (transient EL):}

The champion devices (PS-CzTTM: TPBi) and the reference device (F8BT pristine) were electrically excited by a Keithley 2401 function generator using square voltage pulses with the frequency of $1000 \mathrm{~Hz}$ and a pulse width of 10 $\mu$ s for the on-cycles (forward bias). A reverse bias of $-4 \mathrm{~V}$ was applied to the device after the on-cycle switching off to eliminate the charge accumulation effect. ${ }^{4}$ The instrument response time of the function generator was around 10 ns. The EL emission spectra versus delay time were captured by the same 
ICCD spectrometer used in the PL measurement. The EL intensity as a function of time can be obtained through integrating the EL spectra versus delay time.

Device optimization

Host selection from TPBi, PVK and TFB:

Solutions were prepared from $10 \mathrm{mg} / \mathrm{ml}$ hosts dissolved into toluene.

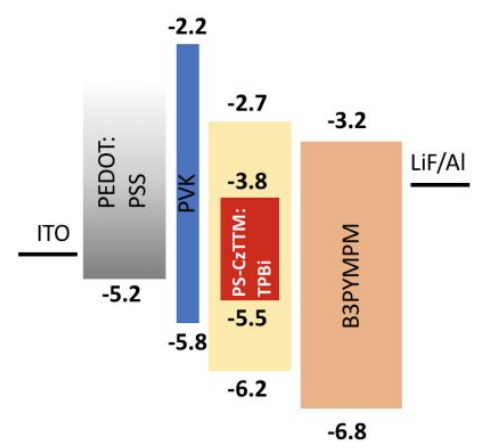

(a)

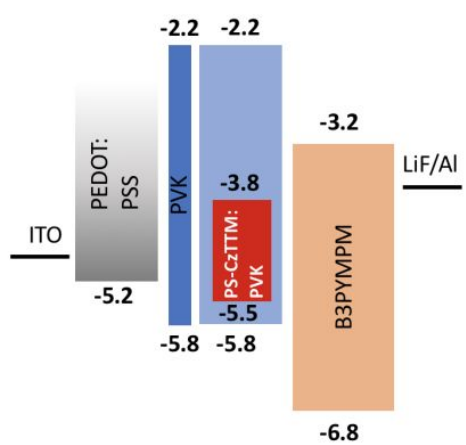

(b)

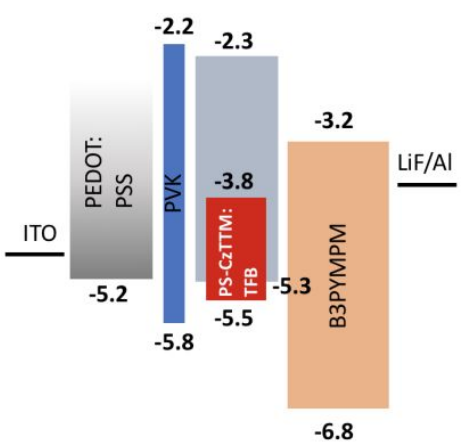

(c) 


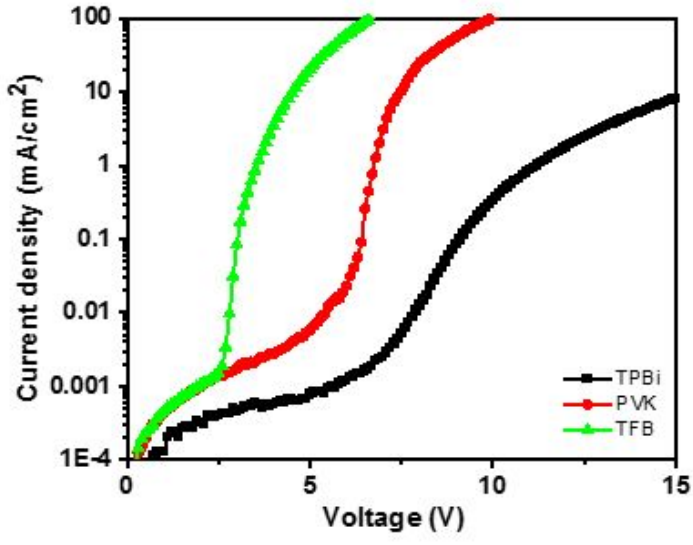

(d)

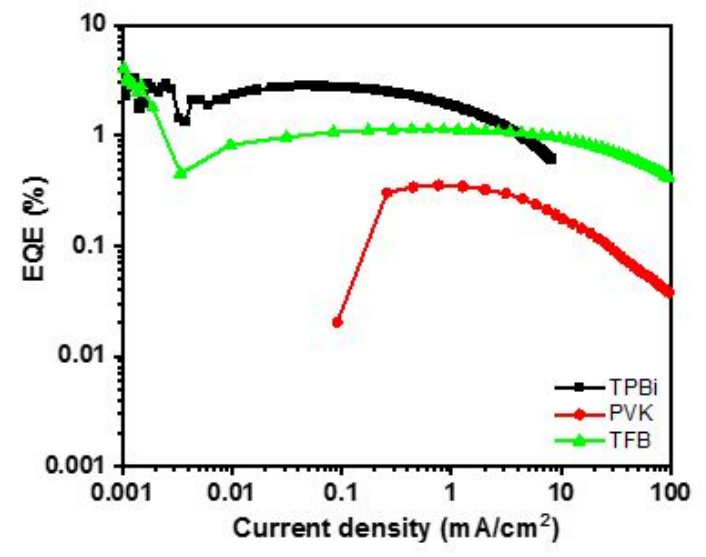

(f)

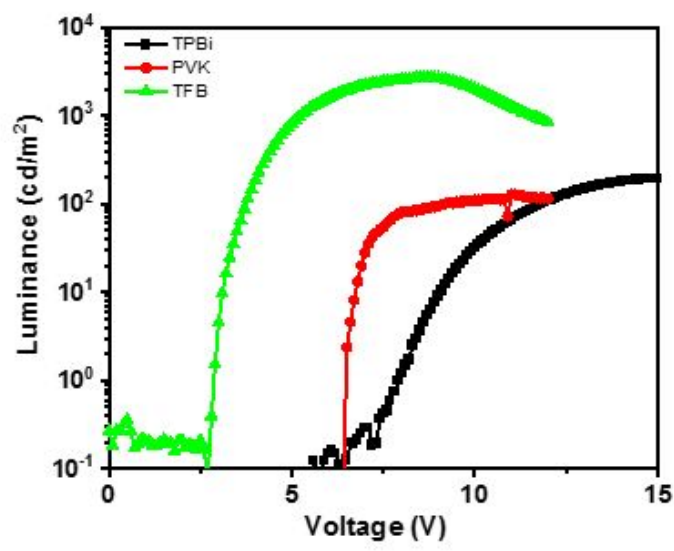

(e)

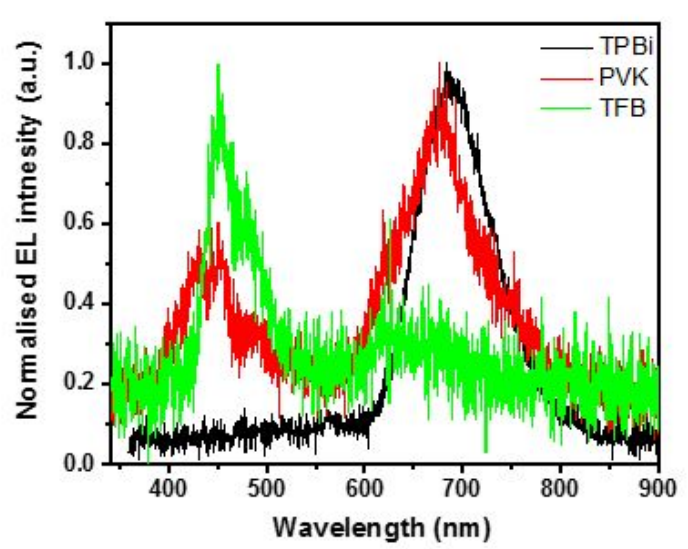

(g)

Figure S1. PS-Cz-TTM device performance with different hosts. Device structures with (a) TPBi; (b) PVK; (c) TFB as the host; (d) current-density vs. voltage; $€$ Luminance vs. voltage; (f) EQE vs. current-density; (g) EL spectra at turn-on voltage

The emission mechanism of the PS-CzTTM champion device (TPBi as the host) is dominated by direct charge trapping. It can be experimentally proved by comparing the EL spectra (Fig. S1g) of devices with other common host matrices (i.e. TFB and PVK) where the emission from the dopant is weaken (in 
PVK) or negligible (in TFB) even at turn-on voltages and disappears as the voltage increases. As the HOMO levels of TFB and PVK are higher or close to that of the PS-CzTTM, direct hole trapping from the host to the dopant is not favorable and the other excitation mechanism, that is, energy transfer (ET), is more feasible theoretically. However, ET is not efficient because the excitons formed in the host undergo direct decay to the ground rather than transferring the energy to the dopant, even though the spectral overlap between the PL of the host and absorption of the dopant is not negligible. TPBi with much lower HOMO offering favorable charge trapping route exhibits a dopant-only emission, indicating that $\mathrm{CT}$ is the favorable and dominant emission mechanism.

\section{Emissive layer (EML) thickness control:}

Film thickness were controlled by varying the concentration of the solution, i.e. $5 \mathrm{mg} / \mathrm{ml}$ for $20 \mathrm{~nm}, 7.5 \mathrm{mg} / \mathrm{ml}$ for $30 \mathrm{~nm}, 10 \mathrm{mg} / \mathrm{ml}$ for $40 \mathrm{~nm}, 15 \mathrm{mg} / \mathrm{ml}$ for 55 $\mathrm{nm}$ and $20 \mathrm{mg} / \mathrm{ml}$ for $70 \mathrm{~nm}$. A $\pm 3 \mathrm{~nm}$ batch-to-batch thickness variation was observed for these solution-processed films. 


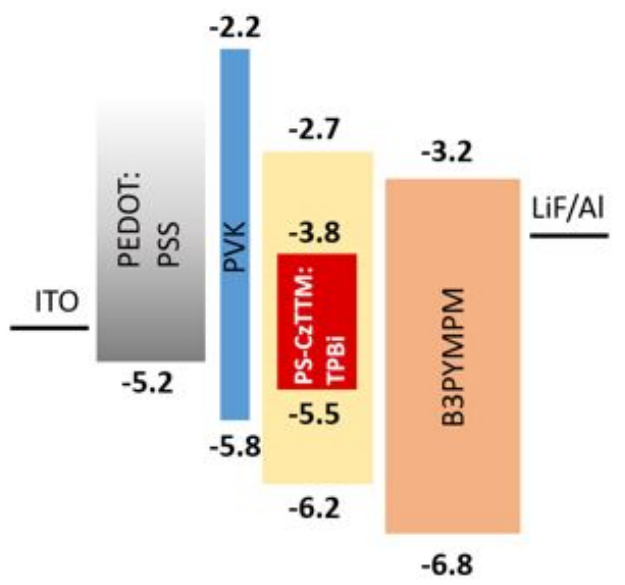

(a)

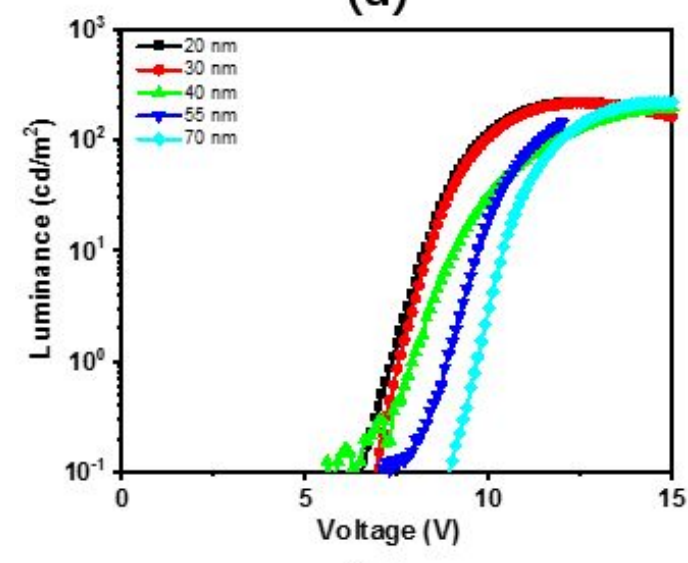

(c)

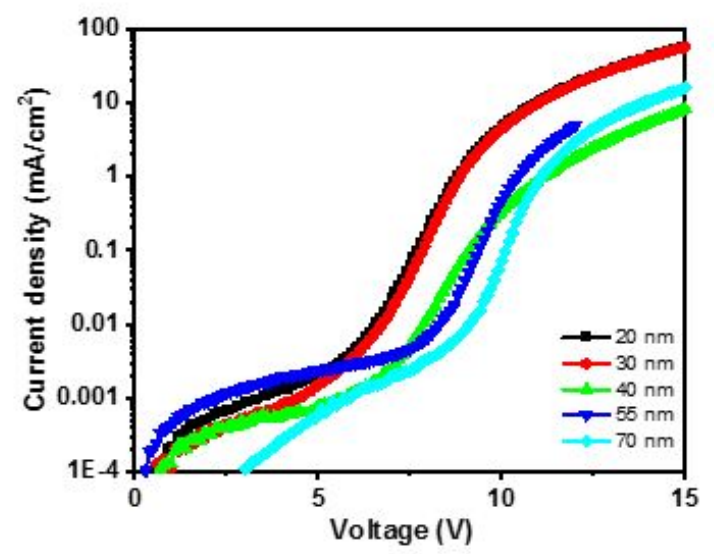

(b)

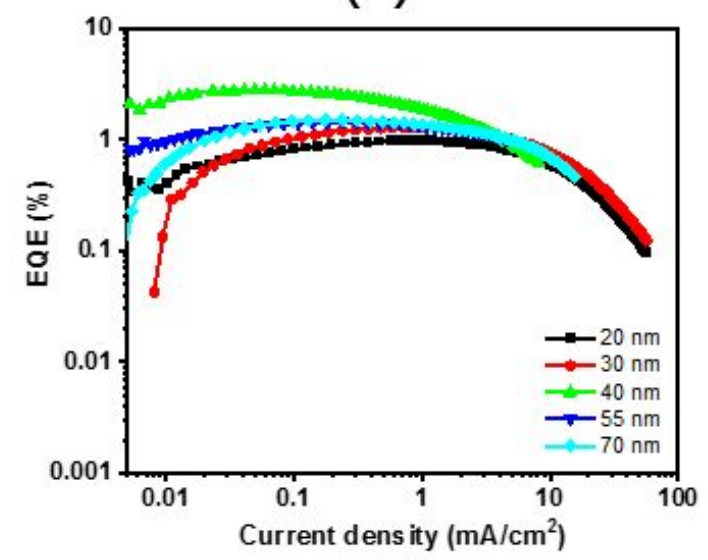

(d)

Figure S2. PS-Cz-TTM device performance with various EML film thickness from 20-70 nm. (a) device structure; (b) current-density vs. voltage; (c) Luminance vs. voltage; (d) EQE vs. current-density.

Table S1. Key performance parameters of PS-CzTTM OLEDs with various film thickness of the EML.

\begin{tabular}{|c|c|c|c|c|c|}
\hline \multirow{2}{*}{ Device } & $V_{O N}$ & $E Q E_{\text {max }}$ & $E Q E$ & $E Q E$ & EL Peak \\
\hline & $(\boldsymbol{V})$ & (\%) & $\left(10 c d m^{-2}, \%\right)$ & $\left(100 c_{d m}^{-2}, \%\right)$ & $(n m)$ \\
\hline $20 \mathrm{~nm}$ & 7.4 & 1.0 & 0.9 & 0.7 & 685 \\
\hline
\end{tabular}




\begin{tabular}{lccccc}
\hline $30 \mathrm{~nm}$ & 7.6 & 1.0 & 0.9 & 0.7 & 685 \\
\hline $40 \mathrm{~nm}$ & 8.0 & 3.0 & 2.9 & 1.1 & 685 \\
\hline $55 \mathrm{~nm}$ & 8.9 & 1.4 & 1.4 & 1.0 & 685 \\
\hline $70 \mathrm{~nm}$ & 9.7 & 1.5 & 1.5 & 1.1 & 685 \\
\hline
\end{tabular}

The turn-on voltage increases as the increase of the thickness of EML (Fig S2). Though thinner EMLs can undergo higher current at the same voltage, their breakdown voltage is lower. Amongst the tried thickness range, $40 \mathrm{~nm}$ EML gives the best charge balance and highest EQE.

Doping concentration control:

All films were prepared from $10 \mathrm{mg} / \mathrm{ml}$ solutions. 


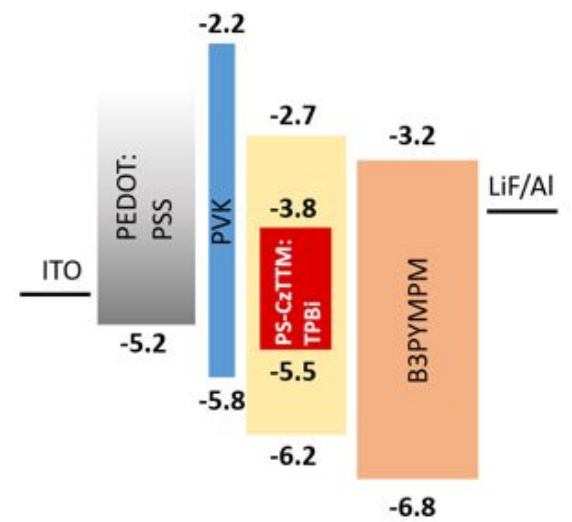

(a)

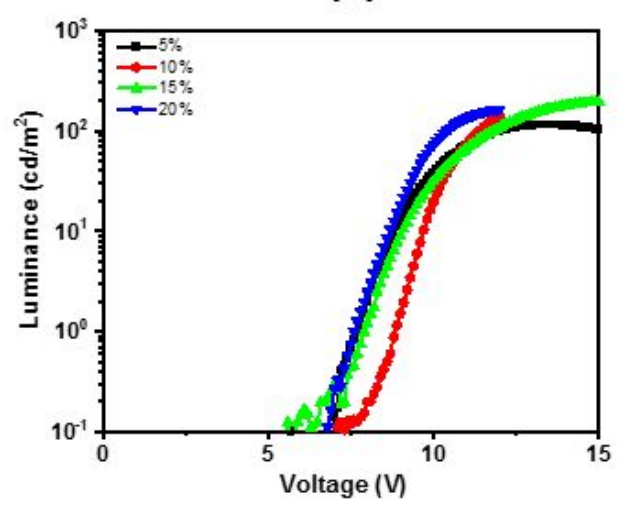

(c)

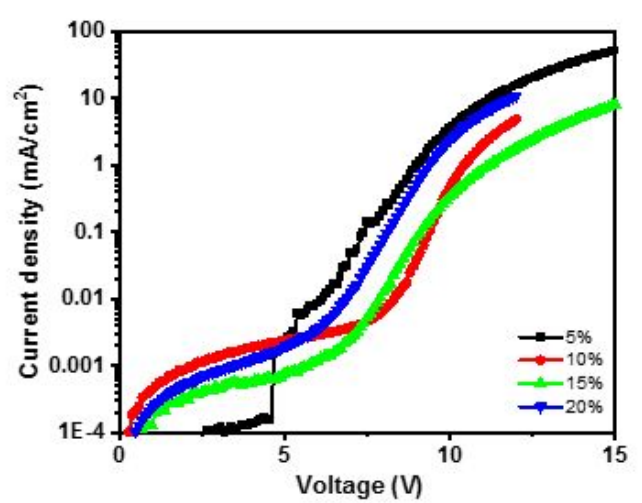

(b)

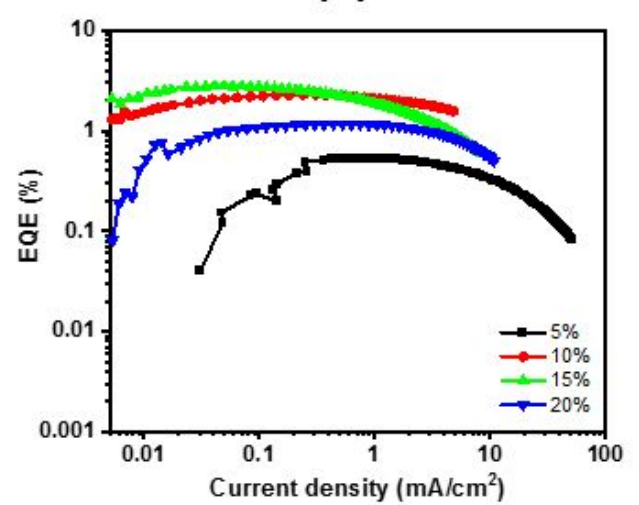

(d)

Figure S3. PS-Cz-TTM device performance with various doping concentration from $5-20 \%$. (a) device structure; (b) current-density vs. voltage; (c) Luminance vs. voltage; (d) EQE vs. current-density.

Table S2. Key performance parameters of PS-CzTTM OLEDs with various concentrations.

\begin{tabular}{lllll}
\hline$V_{O N}$ & $E Q E_{\text {max. }}$ & EQE & EQE & ELPeak
\end{tabular}

Device

(V) $\quad(\%) \quad\left(10 c d m^{-2}, \%\right) \quad\left(100 c d m^{-2}, \%\right) \quad(n m)$

$\begin{array}{llllll}5 \% & 7.8 & 0.5 & 0.5 & 0.2 & 685\end{array}$




\begin{tabular}{llllll}
\hline $10 \%$ & 8.4 & 2.8 & 2.8 & 1.5 & 685 \\
\hline $15 \%$ & 8.0 & 3.0 & 2.9 & 1.1 & 685 \\
\hline $20 \%$ & 7.8 & 1.2 & 1.2 & 0.9 & 685 \\
\hline
\end{tabular}

In terms of doping concentration, devices with $10-15 \%$ of PS-CzTTM in TPBi show the best performance and thus $10-15 \%$ is believed to be the critical concentration to ensure enough exciton generation and avoiding aggregation quenching at the same time (Fig. S3 and Tab. S2).

Structure modification:

Champion structure:

ITO/PEDOT:

PSS/PVK/EML/B3PYMPM/LIF/Al;

W/o(without) PVK:

ITO/PEDOT: PSS/EML/B3PYMPM/LiF/Al;

РОT2T

electron-transporting

layer:

ITO/PEDOT:

PSS/PVK/EML/POT2T/LIF/AI 


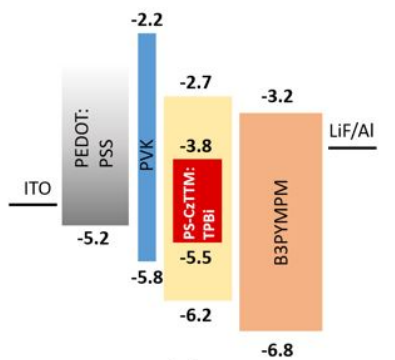

(a)

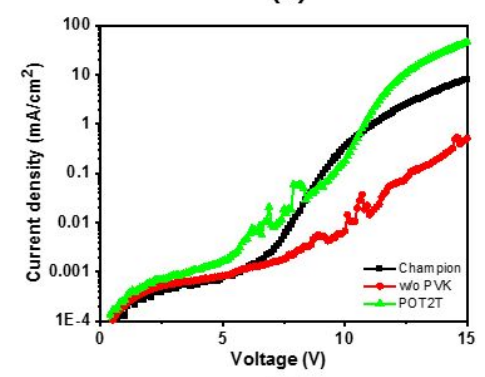

(d)

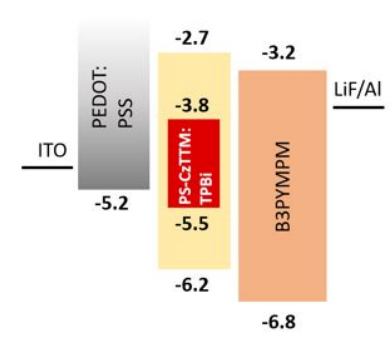

(b)

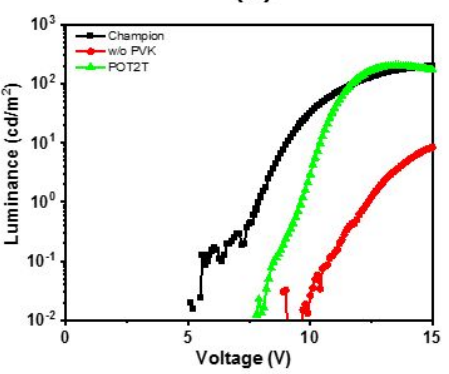

(e)

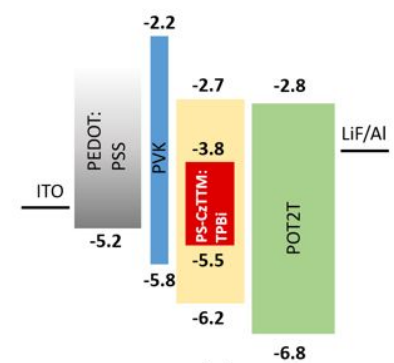

(c)

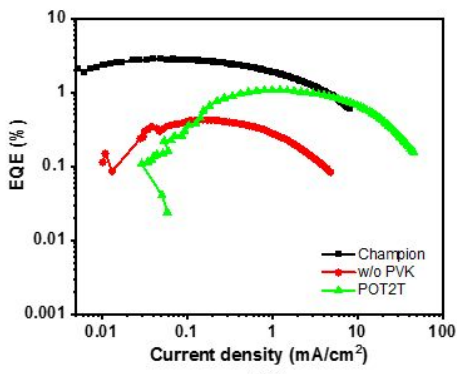

(f)

Figure S4. PS-Cz-TTM device performance in different device structure. (a) champion device structure; (b) device structure without PVK as an extra HTL; (c) device structure using POT2T as the ETL; (d) current-density vs. voltage; (e) Luminance vs. voltage; (f) EQE vs. current-density.

Table S3. Key performance parameters of the champion OLED and other structures.

$$
V_{\text {ON }} \text { EQE }_{\text {max. }} \quad \text { EQE } \quad \text { EQE } \quad \text { ELPeak }
$$

Device

$$
\text { (V) (\%) } \quad\left(10 \mathrm{cdm}^{-2}, \%\right) \quad\left(\mathbf{1 0 0} \mathrm{cdm}^{-2}, \%\right) \quad(\mathrm{nm})
$$

$\begin{array}{llllll}\text { Champion } & 8.0 & 3.0 & 2.9 & 1.1 & 685\end{array}$

$\begin{array}{llllll}\text { w/o PVK } & 12.4 & 0.4 & 0.2 & - & 690\end{array}$

$\begin{array}{llllll}\text { РОT2T } & 9.7 & 1.1 & 0.9 & 0.8 & 685\end{array}$


The champion structure gives much better charge balance, higher EQE and less significant roll-off effect (Tab. S3).

\section{REFERENCES}

(1) De Mello, J. C.; Wittmann, H. F.; Friend, R. H. An Improved Experimental Determination of External Photoluminescence Quantum Efficiency. Adv. Mater. $1997,9,230-232$.

(2) H. Guo et al. High Stability and Luminescence Efficiency in Donor-Acceptor Neutral Radicals Not Following the Aufbau Principle. Nat. Mater. 2019, 18, $977-$ 984.

(3) Conaghan, P. J.; Menke, S. M.; Alexander, S.; Jones, S. T. E.; Pearson, A.

J.; Emrys, W.; Bochmann, M.; Greenham, N. C. Efficient Vacuum-Processed Light-Emitting Diodes Based on Carbene - Metal - Amides. Adv. Mater. 2018, 30, 1802285.

(4) Di, D.; Romanov, A. S.; Yang, L.; Richter, J. M.; Rivett, J. P. H.; Jones, S.; Thomas, T. H.; Jalebi, M. A.; Friend, R. H.; Linnolahti, M.; Bochmann, M.;

Credgington, D. High-Performance Light-Emitting Diodes Based on CarbeneMetal-Amides. Science. 2017, 356, 159-163. 